Wenn nun auch der Inhalt der obigen Schrift vollkommen sich selbst erklärt, so möchte ich doch auf zwei Punkte aufmerksam machen.

Einmal auf die scharfe Gegenüberstellung der Ansichten der Mathematiker und der Naturforscher und ferner, dass Ibn al Haitam die Bedeutung der Lichtstrahlen unabhängig ron den Sehstrahlen gar nicht als etwas besonderes hin. stellt. In der That war diese Auffassung des Lichtes, wie ich bei einer späteren Gelegenheit zeigen werde, im elften Jahrhundert im Orient vielfach vertreten.

XII. ,Die Kölner Luftpumpe v. J. 164166; eine historische Notiz von Dr. G. Berthold in Ronsilorf.

„Die Luftpumpe wurde um das Jahr 1650 von O. von Guericke erfunden", heisst es in den landläufigen Compendien. "Das eigentliche Jahr der Erfindung ist unbekannt," setzte jedoch bereits Lichtenberg hinzu. $\mathrm{Zu}$ letzterem Resultate ist auch Hr. Gerland gelangt'), welcher neuerdings sich vielfach, wiewohl vergeblich, in dieser Sache bemüht hat. Im Gegensatz zu dem vorsichtigen Verhalten Hrn. Gerland's stellt Hr. Zerener mit grosser Emphase die Behauptung $\left.\operatorname{auf}^{2}\right)$, „die Entdeckung der Luftleere, Erfindung der Luftpumpe und anderer anschliessender Apparate sind aber ganz entschieden vor das Jahr 1646 zu datiren," ohne im Stande zu sein, auch nur ein einziges authentisches Document beizubringen. Seine ganze Beweisführung läuft vielmehr darauf hinaus, es sei unwahrscheinlich, dass Guericke, mit Geschäften überbäuft und zu dem in den Jahren 1646 bis 1660 meistens von seiner Vaterstadt abwesend, die

1) Bericht über die wissensch. Apparate auf der Londoner internationalen Ausstellung i. J. 1876. Braunschweig 1878, p. 33. - Beiträge zur Geschichte der Physik. Halle 1882. Fol. p. 5. Anm. 1. - Der leere Raum, die Constitution der Körper u. der Aether. Berlin 1883, p.37. Anm.3.

2) Historisches Nachwort, beigefügt einer Reimpression eines Theiles von Otto von Guericke's Experimenta nova (ut vocantur) Magdeburgica. Leipzig, W. Drugulin. 18ş1. Fol. p. VI. 
nöthige Musse gehabt habe, in dieser Zeit seine physikalischen Untersuchungen anzustellen, seine Entdeckungen seien also vor diese Jahre zu setzen. Einer ähnlichen Argumentation Biedersee's gegenüber hatte bereits Hoffmann bemerkt ${ }^{1}$ ): "so möchte sich mit gutem Grunde dagegen einwenden lassen, dass diese Missionen 0 . v. Guericke nicht fortwährend von Magdeburg entfernt bielten, dass er nach Beendigung der einen nicht sofort eine andere übernehmen musste, sondern bald längere, bald kürzere Zeit (einmal über anderthalb, ein anderes mal beinahe vier volle Jahre) wieder in seiner Vaterstadt verweilt, und dass seine Geschäfte ihm da Musse genug übrig liessen, sich mit seinen Lieblingsstudien $\mathrm{zu}$ beschäftigen. $\mathrm{Ja}$ wir dürfen wohl, da einmal die Neigung für die Naturwissenschaften und für mechanische Arbeiten in ihm erwacht war, als ausgemacht annehmen, dass er sich gleich so vielen anderen Männern während seiner ganzen Lebenszeit, freilich mit längeren oder kürzeren Unterbrechungen, diesen Studien und Beschäftigungen hingegeben habe."

Eine nüchterne Prüfung der Sachlage ergibt nun, dass unser Wissen sich vollständig auf das beschränkte, was Otto von Guericke selbst in seinem Werke berichtet; er schreibt nämlich in der Vorrede ${ }^{2}$ ), durch die Streitigkeiten der Philosophen über den leeren Raum veranlasst, habe er, um der Sache auf den Grund zu kommen, nachdem er die nöthige Musse erlangt hatte, eine experimentelle Prüfung versucht. Dies sei von ihm auf verschiedene Weise versucht, auch sei sein Bemühen nicht vergeblich gewesen, sodass ihm die Erfindung einiger Maschinen gelang, welche geeignet waren, jenen stets geleugneten leeren Raum zu demonstriren. Später (postea) auf dem Reichstage zu Regensburg (1654) habe er auf Wunsch einiger Liebhaber solcher Sachen, welche von den erwähnten Experimenten gehöxt hatten, seine Versuche vorgeführt. Im Jahre 1657 habe dann C. Schott die Luftpumpe nebst den betreffenden, ron letzterem als "Magdeburger" benannten Versuchen durch den Druck bekannt gemacht. Er (Guericke) selbst habe niemals im

1) Otto von Guericke, etc. Ein Lebensbild. Von Fr. W. Hoffmann. Herausgegeben von $\}$. O. Opel. Magdeburg 1874. p. 203.

2) Otto von Guericke, Experimenta nova (ut vocantur) Magdeburgica de Vacao Spatio ete. Amstelodami 1672. Fol. Praefatio (p. X). 
Sinne gehabt, irgend etwas von diesen Sachen drucken zu lassen, doch hätten die widersprechenden Urtheile über den leeren Raum ihn schliesslich veranlasst, seinen vollständigen Tractat de spatio vacuo, der bereits am 14. März 1663 beendet gewesen sei, herauszugeben.

Aus Guericke's eigenem Berichte ist also zu entnehmen, dass die Erfindung der Luftpumpe jedenfalls vor das Jahr $1654 \mathrm{zu}$ setzen ist, ob dieselbe aber kurz vor dieses Jahr fällt, oder ob der Termin weiter zurück liegt, darüber besagen unsere Quellen nichts, ausgenommen allerdings eine einzige, sehr späte Literaturangabe, welche diesen Termin mindestens bis zu dem Jahre 1651 zurückschiebt. Es ist dies die vielberufene Angabe Muncke's, welcher i. J. 1831 in Gehler's physikalischem Wörterbuche berichtet ${ }^{1}$ ): „Uebrigens hat Otto v. Guericke mehrere (sc. Luftpumpen) verfertigen lassen, namentlich eine, welche er schon 1651 dem Magistrate in Cöln zum Geschenke machte. S. Hindenburg's Magaz. Heft X p. 120." Diese Angabe Muncke's hat eine sehr verschiedene Aufnahme bei den Autoren gefunden. Hr. Zerener nimmt sie ohne weiteres als gesichert an und baut seine Schlüsse darauf. ${ }^{2}$ ) Reservirter zeigt sich Hoffmann, indem er zugleich fragt: „Was bestimmte ihn (sc. Guericke), den Vätern dieser Stadt eine derartige Auszeichnung zu Theil werden zu lassen? Geschah dies auf ihren Wunsch oder aus eigenem Antriebe? Und wenn das letztere der Fall war, hatte er irgend welche Verbindlichkeiten gegen sie? Oder waren sie durch Bande der Freundschaft mit ihm verknüpft? Alles das sind Fragen, auf welche keine Antwort ertheilt werden kann." 3 ) Hr. Gerland endlich verbält sich vollständig ablehnend dagegen, indem er Muncke's Citat einfach für falsch erklärt.4)

1) Gehler's physikalisches Lexicon. Leipzig 1831. 6. p. 527. Anm. 1.

2) l. c. p. 6 .

3) 1. c. p. 211.

4) Bericht ete. p. 33. - Der leere Raum etc. p. 37. Anm. 3. An letzterem Orte schreibt Hr. Gerland: "Die von Muncke in Gehler's physikal. Lexicon, 6. p. 527 angeführten Citate sind falsch." Dies ist jedoch ein Irrthum; wie es sich mit dem Citat in Betreff der Kölner Luftpumpe verhält, werden wir weiter oben sehen. Das andere Citat in Betreff der Berliner Luftpumpe ist vollkommen richtig; in dem von Muncke citirten Werke, Nachrichten von dem Leben und den Erfindungen der berühmtesten Mathematiker. In alphabetischer Ordnung. Erster Theil, welcher die bis jetzt bereits Verstorbenen enthált. Münster, bei Philipp Heinrich Perrenon $1788 . \mathrm{kl} .8^{\circ}$. p. 121 
Wenn sich nun auch das Citat als unrichtig erwies, so musste es doch einen eigenen Reiz gewähren, der Angabe selbst näher zu treten und zuzusehen, ob sich auch diese als falsch erwiese, oder ob sie sich verificiren lasse. Meine eigenen, darauf bezüglichen Nachforschungen führten nun alsbald zu dem gewünschten Resultate. Ich machte nämlich zunächst ausfindig, dass Muncke seine Angabe aus Busch, Handbuch der Erfindungen, entlehnt hat, und dass es sich bei dem Citate lediglich um einen Schreibfehler Muncke's handelt. G. C. Busch berichtet nämlich 1): „In Cöln befindet sich eine Luftpumpe, die Guericke selbst machte und im Jahre 1651 dem Magistrat zum Geschenk überschickte. Archiv für die reine und angewandte Mathematik von Hindenburg, 10. Heft, p. 132." Also. nicht das Magazin Hindenburg's, wie Muncke citirt, sondern das Archiv war die richtige Quelle, wie ein Nachschlagen sofort zeigte. Eine Vergleichung der Urquelle in Hindenburg's Archiv ergab nun aber eine höchst überraschende Abweichung, indem hier nicht, wie bei Busch (und nach diesem bei Muncke) das Jahr 1651, sondern das Jahr 1641 angegeben ist.

Im zehnten Hefte von Hindenburg's Archiv sind einige Briefe von Christian Kramp v. J, 1799 an Hindenburg abgedruckt. Kramp war in diesem Jahre zunächst als Professor der Mathematik, später als Professeur de Chimie ot de Physique expérimentale á l'Ecole centrale du Département de la Roer in Cöln angestellt worden. Er war eifrig mit der Ordnung und Completirung des physikalischen Cabinets beschäftigt und so glücklich gewesen, für die Centralschule das schöne physikalische Cabinet des Professors Schurer in Strassburg zu erwerben. Von Strassburg aus,

(Artikel Guerike) heisst es: „Die Luftpumpe des Otto von Guerike ist noch zu Berlin auf der königlichen Bibliothek zu sehen; ihre Einrichtung ist von der jetzt gewöhnlichen sehr verschieden und gewissermassen noch unvollkommen."

1) Handbuch der Erfindungen. Eisenach 1816. Th. VIII. p. 223. Uebrigens findet sich auch bei Busch ein Druckfehler, indem dort Hindenburg's Archiv, Heft X p. 182 steht, während es p. 232 heissen muss. - Höchst auffallend ist es, dass Hoffmann, l. c. p. 211. Anm. 3, obschon er Gehler's phys. Lex. citirt, doch Hindenburg's Archiv druckt (nicht Magazin, wie im Gehler steht), und gleichfalls, wie Busch, p. 132 setzt, während im Gehler p. 120 steht. 
wohin sich Kramp begeben batte, um das Einpacken und den Transport der Schurer'schen Sammlung zu besorgen, schreibt er nun in einem d. 20. Floréal VII. datirten Briefe: "In Cölln selbst ist bereits eine artige Sammlung physikalischer Sachen: eine Querickische Luftpumpe, von ihm selbst gemacht und im Jahre 1641 dem Magistrate von Cölln zum Präsent geschickt; eine neuere Nolletische, eine ganz neue Smeatonische, nebst dazu gehörigem, vollständigem Apparate. . . Kurz wir haben nunmehr eine der vollständigsten Sammlungen, die in der Republique anzutreffen." 1 )

Diese Mittheilung Kramp's ist geeignet, unser höchstes Interesse zu erwecken. Findet sich die als i. J. 1799 in Cöln noch vorhanden bezeichnete Luftpumpe noch gegenwärtig dort? Welches sind die Beweise für ihre Echtheit? Und vor allem, lässt sich authentisch nachweisen, dass diese Luftpumpe bereits i. J. 1641 von Guericke nach Cöln geschickt wurde? ${ }^{2}$ ) Das sind Fragen, die sich Jedem sofort aufdrängen. Hr. Dr. Becker, Oberbürgermeister von Cöln, hatte die ausserordentliche Gefälliglreit, auf meine Bitte an Ort und Stelle Nachforschungen vorzunehmen. Es gelang jedoch nicht, von der Luftpumpe auch nur eine Spur aufzufinden; Hr. Becker rermuthet, dass die Luftpumpe in der französischen Zeit abhanden gekommen ist; in dem amtlichen Verzeichniss der nach Paris entführten Sachen ist sie übrigens nicht mit aufgefübrt. $O b$ noch ein altes Verzeichniss der physikalischen Sammlung der ehemaligen Centralschule zu Cöln vorhanden ist, und ob sich im Stadtarchiv von Cöln Schriftstücke ron Guericke oder sonstiges Actenmaterial

1) C. F. Hindenburg, Archiv der reinen und angewandten Mathematik. Leipzig, 1799. Zehntes Heft. p. 232.

2) Ich will hier ausdrücklich bemerken, dass sich ein Druckfehlerverzeichniss des zehnten Heftes von Hindenburg's Archiv nicht findet, anch nicht in dem 11. Hefte, für welches ein solches (für Heft $X$ ) versprochen war; ebenso wenig findet sich bei Busch ein Druckfehlerverzeichniss. Es bleibt unaufgeklärt, ob es sich bei Busch in Bezug auf das Jahr um einen Druckfehler handelt, oder ob Busch willkürlich die Jahreszahl geändert hat, um dieselbe conformer mit dem gewöhnlich als Zeitpunkt der Erfindung der Luftpumpe angenommenen Jahre $1650 \mathrm{zu}$ machen. - Die Möglichkeit, dass es sich in Bezug auf die Jahreszahl 1641 bei Kramp, ganz abgesehen von einem etwaigen Druck- oder Schreibfebler, um einen Gedächtnissfehler handeln kann, ist natürlich nicht ansgeschlossen, zumal da Kramp von Strassburg und nicht von Cöln aus seine Mittheilung an Hindenburg machte. 
vorfindet, welches zur Aufklärung der Sache dienen könnte, darüber konnte ich leider keine Auskunft erhalten. $\left.{ }^{1}\right)$

Unter so bewandten Umständen bleibt nichts anderes übrig, als die Angabe Kra mp's vorläufig einfach zu registriren. Als ein historisches Factum von unzweifelhafter Sicherheit vermögen wir dieselbe nicht $\mathrm{zu}$ betrachten, da die authentischen Beweise für ihre Richtigkeit fehlen. Wir schliessen, indem wir den Ausspruch Lichtenberg's dahin modificiren: Es ist nicht nur das eigentliche Jahr der Erfindung der Luftpumpe durch Otto von Guericke vollständig unbekannt, sondern sogar der ungefähre Zeitpunkt der Erfindung hat bis jetzt nicht festgestellt werden können.

\section{Bemerkung zu der Abhandlung}

des Hrn. Christiansen: ,Einige Versuche über die Wärmeleitung; von A. Winkelmann.

Hr. Christiansen hat unter dem obigen Titel einige Versuche mitgetheilt ${ }^{2}$ ), welche $u$. a. auch die Abhängigkeit der Wärmeleitung der Luft von der Temperatur bestimmen. Die Methode, deren sich Hr. Christiansen bediente, war die folgende: Drei runde, gleich grosse Kupferplatten waren durch je drei kleine Glasstuickchen voneinander getrennt; die untere Platte wurde durch kaltes, die obere durch warmes Wasser auf einer constanten Temperatur gehalten, und die Temperatur der drei Platten durch Thermometer bestimmt. Sind die Platten gleich weit $(e)$ voneinander entfernt, wie es bei den Versuchen mit Luft der Fall war, so hat man folgende Gleichung (5) nach Hrn. Christiansen:

Hier bezeichnet:

$$
\begin{gathered}
S . k\left(1+\frac{T_{1}+T_{2}}{2} \ll\right) \frac{T_{1}-T_{2}}{e}-S k\left(1+\frac{T_{2}+T_{3}}{2} \alpha\right) \frac{T_{3}-T_{3}}{e} \\
=h . A \cdot\left(T_{3}-T_{0}\right) .
\end{gathered}
$$

$S$ die Grundfläche einer Platte,

$k$ die Leitungsfähigkeit der Luft bei $0^{0}$,

$h$ die äussere Wärmeleitungsfähigkeit des Kupfers,

$A$ die cylindrische Oberfläche der mittleren Platte,

1) Fr. Dr. Becker theilt mir mit, dass der Stadtarchivar gegenwärtig mit anderen Arbeiten vollauf beschäftigt und daher jetzt und in nächster Zeit nicht in der Lage sei, die bezüglichen Nachforschungen vorzunehmen. - Ich behalte mir vor, zu gelegener Zeit selbst diese Nachforschungen im Stadtarchiv auszuführen.

2) Christiansen, Wied. Ann. 14. p. 28.1881. 